\title{
Multigrid Methods for Elliptic Obstacle Problems on 2D Bisection Grids
}

\author{
Long Chen ${ }^{1}$, Ricardo H. Nochetto ${ }^{2}$, and Chen-Song Zhang ${ }^{3}$ \\ 1 Department of Mathematics, University of California at Irvine. \\ chenlong@math.uci.edu \\ 2 Department of Mathematics, University of Maryland at College Park. \\ rhn@math.umd.edu \\ 3 Department of Mathematics, The Pennsylvania State University. \\ Corresponding author. zhangcs@psu.edu
}

\section{Introduction}

In this paper, we develop and analyze an efficient multigrid method to solve the finite element systems from elliptic obstacle problems on two dimensional adaptive meshes. Adaptive finite element methods (AFEMs) based on local mesh refinement are an important and efficient approach when the solution is non-smooth. An optimality theory on AFEM for linear elliptic equations can be found in Nochetto et al. (2009). To achieve optimal complexity, an efficient solver for the discretization is indispensable.

The classical projected successive over-relaxation method by Cryer (1979) converges but the convergence rate degenerates quickly as the mesh size approaches zero. To speed up the convergence, different multigrid and domain decomposition techniques have been developed (see the monograph by Kornhuber (1997) and the recent review by Graser and Kornhuber (2009).) In particular, the constraint decomposition method by Tai (2003) is proved to be convergent linearly with a rate which is almost robust with respect to the mesh size in $\mathbb{R}^{2}$; but the result is restricted to uniformly refined grids.

We shall extend the algorithm and theoretical results by Tai (2003) to an important class of adaptive grids obtained by newest vertex bisections; thereafter we call them bisection grids for short. This is new according to Graser and Kornhuber (2009): the existing work assumes quasi-uniformity of the underlying meshes. Based on a decomposition of bisection grids due to Chen et al. (2009), we present an efficient constraint decomposition method on bisection grids and prove an almost uniform convergence

$$
\mathcal{J}\left(u^{k}\right)-\mathcal{J}\left(u^{*}\right) \leq C\left(1-\frac{1}{1+\left|\log h_{\min }\right|^{2}}\right)^{k},
$$


where $\mathcal{J}(u)=\int_{\Omega}\left(\frac{1}{2}|\nabla u|^{2}-f u\right) \mathrm{d} x$ is the objective energy functional, $u^{k}$ is the $k$-th iteration and $u^{*}$ is the exact solution of the constrain minimization problem, $h_{\text {min }}=\min _{\tau \in \mathcal{T}} \operatorname{diam}(\tau)$ and the grid $\mathcal{T}$ is obtained by bisections from a suitable initial triangulation $\mathcal{T}_{0}$.

\section{Constraint Decomposition Methods}

The subspace correction framework Xu (1992) has been extended to nonlinear convex minimization problems by Tai and $\mathrm{Xu}$ (2002). This technique has also been applied to develop domain decomposition and multigrid methods for obstacle problems in Badea et al. (2003); Tai et al. (2002). Furthermore, a constraint decomposition method (CDM) was introduced and proved to have a contraction factor which is almost independent of mesh size by Tai (2003). In this section, we briefly review the CDM for obstacle problems.

Let $\mathbb{V} \subset H_{0}^{1}$ be a finite dimensional Hilbert space and $\mathcal{J}: \mathbb{K} \rightarrow \mathbb{R}$ be a convex functional defined over the convex set $\mathbb{K} \subset \mathbb{V}$. We consider the energy minimization problem

$$
\min _{v \in \mathbb{K}} \mathcal{J}(v) .
$$

In this paper, for simplicity, we only consider the case

$$
\mathcal{J}(u):=\int_{\Omega}\left(\frac{1}{2}|\nabla u|^{2}-f u\right) \mathrm{d} x \quad \text { and } \quad \mathbb{K}:=\{v \in \mathbb{V} \mid v \geq 0\},
$$

where $\Omega \subset \mathbb{R}^{2}$ is a polygonal domain, $\mathcal{T}$ is a conforming triangulation of $\Omega$, $\mathbb{V}=\mathbb{V}(\mathcal{T})$ is the continuous piecewise linear finite element space over $\mathcal{T}$. Let $\|\cdot\|$ be the norm associated to the energy $\mathcal{J}$. For our choice of $\mathcal{J}$ in (3), the energy norm is $\|u\|=\|\nabla u\|$. The algorithm discussed in this paper can be generalized to problems with more general energies and obstacles.

We decompose the space $\mathbb{V}$ into a sum of subspaces $\mathbb{V}_{i} \subset \mathbb{V}, i=1, \ldots, m$ :

$$
\mathbb{V}=\mathbb{V}_{1}+\cdots+\mathbb{V}_{m}=\sum_{i=1}^{m} \mathbb{V}_{i}
$$

and further decompose the convex set $\mathbb{K}$ as follows

$$
\mathbb{K}=\mathbb{K}_{1}+\cdots+\mathbb{K}_{m}=\sum_{i=1}^{m} \mathbb{K}_{i} \text { with } \mathbb{K}_{i} \subset \mathbb{V}_{i}(i=1, \ldots, m),
$$

where $\mathbb{K}_{i}$ are convex and closed in $\mathbb{V}_{i}$. Then we have the following abstract algorithm of successive subspace correction type.

Algorithm 1 (CDM) Given an initial guess $u^{0} \in \mathbb{K}$.

For $k=0,1, \ldots$, till convergence

Decompose $u^{k}=\sum_{i=1}^{m} u_{i}$, such that $u_{i} \in \mathbb{K}_{i}$; and let $w^{0}=u^{k}$.

For $i=1: m$

End For

$$
w^{i}=w^{i-1}+\operatorname{argmin}_{d_{i}}\left\{\mathcal{J}\left(w^{i-1}+d_{i}\right) \mid d_{i} \in \mathbb{V}_{i} \text { and } u_{i}+d_{i} \in \mathbb{K}_{i}\right\} .
$$

End For

Let $u^{k+1}=w^{m}$. 
It is clear that each iteration $w^{i}(i=1, \ldots, m)$ stays in the feasible set $\mathbb{K}$ due to (5). The linear convergent rate of Algorithm 1 has been established by Tai (2003) under the following assumptions:

Assumption 2 (Assumptions on Decomposition) We assume that

1. Nonlinear Stability: For any $u, v \in \mathbb{K}$, there exist a constant $C_{1}>0$ and decompositions $u=\sum_{i=1}^{m} u_{i}, v=\sum_{i=1}^{m} v_{i}$ with $u_{i}, v_{i} \in \mathbb{K}_{i}$ such that

$$
\left(\sum_{i=1}^{m}\left\|u_{i}-v_{i}\right\|^{2}\right)^{\frac{1}{2}} \leq C_{1}\|u-v\| ;
$$

2. Nonlinear Strengthened Cauchy-Schwarz: There exists $C_{2}>0$ such that

$$
\sum_{i, j=1}^{m}\left|\left\langle\mathcal{J}^{\prime}\left(w_{i j}+v_{i}\right)-\mathcal{J}^{\prime}\left(w_{i j}\right), \widetilde{v}_{j}\right\rangle\right| \leq C_{2}\left(\sum_{i=1}^{m}\left\|v_{i}\right\|^{2}\right)^{\frac{1}{2}}\left(\sum_{j=1}^{m}\left\|\widetilde{v}_{j}\right\|^{2}\right)^{\frac{1}{2}},
$$

for any $w_{i j} \in \mathbb{V}, v_{i} \in \mathbb{V}_{i}$, and $\widetilde{v}_{j} \in \mathbb{V}_{j}$.

Theorem 3 (Convergence Rate of CDM) If Assumption 2 is satisfied, then Algorithm 1 converges linearly and

$$
\frac{\mathcal{J}(w)-\mathcal{J}\left(u^{*}\right)}{\mathcal{J}(u)-\mathcal{J}\left(u^{*}\right)} \leq 1-\frac{1}{\left(\sqrt{1+C_{0}}+\sqrt{C_{0}}\right)^{2}},
$$

where $u^{*}$ is the solution of (2) and $C_{0}=2 C_{2}+C_{1}^{2} C_{2}^{2}$.

\section{A Constraint Decomposition on Bisection Grids}

In this section, we construct subspace decompositions of the linear finite element space $\mathbb{V}$, as well as a constraint decomposition of $\mathbb{K}$, on a bisection grid $\mathcal{T}$. Our new algorithm is based on a decomposition of bisection grids introduced in Chen et al. (2009); see also Xu et al. (2009).

For each triangle $\tau \in \mathcal{T}$, we label one vertex of $\tau$ as the newest vertex and call it $V(\tau)$. The opposite edge of $V(\tau)$ is called the refinement edge and denoted by $E(\tau)$. This process is called labeling of $\mathcal{T}$. Given a labeled initial grid $\mathcal{T}_{0}$, newest vertex bisection follows two rules:

1. a triangle (father) is bisected to obtain two new triangles (children) by connecting its newest vertex with the midpoint of its refinement edge;

2. the new vertex created at the midpoint of the refinement edge is labeled as the newest vertex of each child.

Therefore, refined grids $\mathcal{T}$ from a labeled initial grid $\mathcal{T}_{0}$ inherit labels according to the second rule and the bisection process can thus proceed. We define $\mathbb{C}\left(\mathcal{T}_{0}\right)$ as the set of conforming triangulations obtained from $\mathcal{T}_{0}$ by newest vertex 
bisection(s). It can be easily shown that all the descendants of a triangle in $\mathcal{T}_{0}$ fall into four similarity classes and hence any triangulation $\mathcal{T} \in \mathbb{C}\left(\mathcal{T}_{0}\right)$ is shape-regular.

Let $\mathcal{T}$ be a labeled conforming mesh. Two triangles sharing a common edge are called neighbors to each other. A triangle $\tau$ has at most three neighbors. The neighbor sharing the refinement edge of $\tau$ is called the refinement neighbor and denoted by $F(\tau)$. Note that $F(\tau)=\varnothing$ if $E(\tau)$ is on the boundary of $\Omega$. Although $E(\tau) \subset F(\tau)$, the refinement edge of $F(\tau)$ could be different than $E(\tau)$. An element $\tau$ is called compatible if $F(F(\tau))=\tau$ or $F(\tau)=\varnothing$. We call a grid $\mathcal{T}$ compatibly labeled if every element in $\mathcal{T}$ is compatible and call such a labeling of $\mathcal{T}$ a compatible labeling.

For a compatible element $\tau$, its refinement edge $e$ is called a compatible edge, and $\omega_{e}=\tau \cup F(\tau)$ is called a compatible patch. By this definition, if $e$ is a compatible edge, $\omega_{e}$ is either a pair of two triangles sharing the same refinement edge $e$ or one triangle whose refinement edge $e$ is on the boundary. In both cases, bisection of triangles in $\omega_{e}$ preserves mesh conformity; we call such a bisection a compatible bisection. Mathematically, we define the compatible bisection as a map $b_{e}: \omega_{e} \rightarrow \omega_{p}$, where $\omega_{p}$ consists of all triangles sharing the new point $p$ introduced in the bisection. We then define the addition $\mathcal{T}+b_{e}:=\left(\mathcal{T} \backslash \omega_{e}\right) \cup \omega_{p}$. For a sequence of compatible bisections $\mathcal{B}=\left(b_{1}, b_{2}, \cdots, b_{m}\right)$, we define

$$
\mathcal{T}+\mathcal{B}:=\left(\left(\mathcal{T}+b_{1}\right)+b_{2}\right)+\cdots+b_{m},
$$

whenever the addition is well defined.
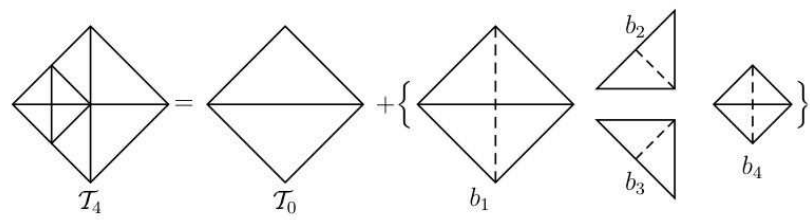

Fig. 1. A decomposition of a bisection grid.

Theorem 4 (Decomposition of Bisection Grids) If $\mathcal{T}_{0}$ is conforming and compatibly labeled, then for any $\mathcal{T} \in \mathbb{C}\left(\mathcal{T}_{0}\right)$, there exists a compatible bisection sequence $\mathcal{B}=\left(b_{1}, b_{2}, \cdots, b_{m}\right)$, such that

$$
\mathcal{T}=\mathcal{T}_{0}+\mathcal{B}
$$

Remark 1. We only give a pictorial demonstration in Fig. 1 to illustrate the decomposition. For the proof of Theorem 4, we refer to Chen et al. (2009); Xu et al. (2009). A practical decomposition algorithm has been developed and implemented by Chen and Zhang (2009). 
Throughout this paper, we will assume that $\mathcal{T} \in \mathbb{C}\left(\mathcal{T}_{0}\right)$ has been decomposed as in (7). We denote the intermediate grids by

$$
\mathcal{T}_{i}:=\left(\left(\mathcal{T}_{0}+b_{1}\right)+b_{2}\right) \cdots+b_{i} \quad i=1, \ldots, m,
$$

and observe that $\mathcal{T}_{i} \in \mathbb{C}\left(\mathcal{T}_{0}\right)$. Let $\mathcal{P}\left(\mathcal{T}_{i}\right)$ denote the set of interior vertices of the triangulation $\mathcal{T}_{i}$. Denote by $\psi_{i, p} \in \mathbb{V}\left(\mathcal{T}_{i}\right)$ the nodal basis function associated with a node $p \in \mathcal{P}\left(\mathcal{T}_{i}\right)$ and by $\omega_{i, p}$ the local patch (i.e. the support of $\left.\psi_{i, p}\right)$. The subspace corresponding to the compatible bisection $b_{i}$, which introduces the new vertex $p_{i} \in \mathcal{P}\left(\mathcal{T}_{i}\right)$, can be written as $\mathbb{V}_{i}:=\operatorname{span}\left\{\psi_{i, p}, p \in \mathcal{P}\left(\mathcal{T}_{i}\right) \cap \omega_{i, p_{i}}\right\}$. To enforce the homogenous Dirichlet boundary condition, we simply set $\mathbb{V}_{i}=$ $\varnothing$ if $p_{i}$ is a vertex on the boundary. Let $\mathbb{V}_{0}=\mathbb{V}\left(\mathcal{T}_{0}\right)$ be the linear space corresponding to the initial mesh $\mathcal{T}_{0}$. Then we have a space decomposition $\mathbb{V}=\sum_{i=0}^{m} \mathbb{V}_{i}$.

Based on this space decomposition, there are infinitely many possibilities to decompose the feasible set $\mathbb{K}$. We do not consider the optimal way to choose such a constraint decomposition. We simply choose

$$
\mathbb{K}=\sum_{i=0}^{m} \mathbb{K}_{i} \text { with } \mathbb{K}_{i}:=\left\{v \in \mathbb{V}_{i} \mid v \geq 0\right\},
$$

and focus on how to decompose $u \in \mathbb{V}$ at each iteration in Algorithm 1. Let $\mathbb{W}_{j}=\sum_{i=0}^{j} \mathbb{V}_{i}, j=1, \ldots, m$. For $i=m, m-1, \ldots, 1$, we first define $I_{i}^{i-1}: \mathbb{W}_{i} \rightarrow \mathbb{W}_{i-1}$ such that

$$
I_{i}^{i-1} v(p)= \begin{cases}\min \left\{v(p), v\left(p_{i}\right)\right\}, & \text { if } p \in \mathcal{P}\left(\mathcal{T}_{i-1}\right) \cap \omega_{i, p_{i}} \\ v(p), & \text { if } p \in \mathcal{P}\left(\mathcal{T}_{i-1}\right) \backslash \omega_{i, p_{i}} .\end{cases}
$$

We then define $Q_{i}: \mathbb{V} \rightarrow \mathbb{W}_{i-1}$ to be $Q_{i}:=I_{i}^{i-1} I_{i+1}^{i} \cdots I_{m}^{m-1}$. Notice that $Q_{i}$ 's are nonlinear operators, i.e. $Q_{i} u-Q_{i} v \neq Q_{i}(u-v)$. Finally we define a decomposition $u=\sum_{i=0}^{m} u_{i}$, with

$$
u_{m}:=u-Q_{m} u, \quad u_{i}:=Q_{i+1} u-Q_{i} u(i=m-1, \ldots, 1), \quad u_{0}=Q_{1} u .
$$

Comparing these with the definitions of $\mathbb{V}_{i}$ and $\mathbb{K}_{i}$, we can easily see that $u_{i} \in \mathbb{K}_{i}$, for $i=0,1, \ldots, m$.

Now we prove the convergence rate of the proposed algorithm.

Lemma 1 (Stability of $Q_{i}$ ). Let $u, v \in \mathbb{V}$. For $i=0,1, \ldots, m$ and any element $\tau \in \mathcal{T}_{i}$, we have

$$
h_{\tau}^{-1}\left\|Q_{i+1} u-Q_{i+1} v\right\|_{L^{2}(\tau)} \leq C\left(1+\left|\log \left(h_{\tau} / h_{\min }\right)\right|\right)^{\frac{1}{2}}\|u-v\|_{H^{1}\left(\omega_{i, \tau}\right)},
$$

where $C$ is a generic constant independent of the meshsize.

Proof. From the definition of $Q_{i}$, for any $u, v \in \mathbb{V}$, we have that

$\left\|Q_{i+1} u-Q_{i+1} v\right\|_{L^{2}(\tau)} \leq C \sum_{p \in \mathcal{P}\left(\mathcal{T}_{i}\right) \cap \tau}\|u-v\|_{L^{\infty}\left(\omega_{i, p}\right)}|\tau|^{\frac{1}{2}} \leq C h_{\tau}\|u-v\|_{L^{\infty}\left(\omega_{i, \tau}\right)}$.

The result then follows directly from the discrete Sobolev inequality between $L^{\infty}$ and $H^{1}$ in two dimensions; see Bramble et al. (1986). 


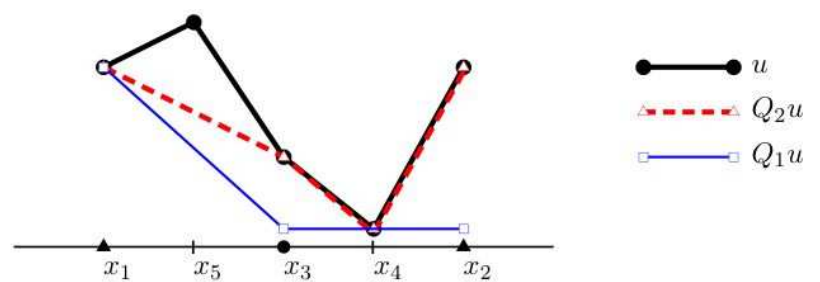

Fig. 2. A one-dimensional example for the decomposition of $u$. Suppose the initial grid $\mathcal{T}_{0}=\left\{\left(x_{1}, x_{3}\right),\left(x_{3}, x_{2}\right)\right\}$. And the final grid $\mathcal{T}$ can be viewed as $\mathcal{T}_{0}+b_{1}+b_{2}$ where $b_{1}$ bisects the element $\left(x_{3}, x_{2}\right)$ and introduces $x_{4}$ and $b_{2}$ bisects $\left(x_{1}, x_{2}\right)$ and introduces $x_{5}$. As we discussed above $\mathcal{T}_{1}=\mathcal{T}_{0}+b_{1}$ and $\mathcal{T}=\mathcal{T}_{2}=\mathcal{T}_{1}+b_{2}$. From the definition of $Q_{i}$, we can easily obtain a decomposition of $u$.

We introduce the generation of elements and compatible bisections. The generation of each element in the initial grid $\mathcal{T}_{0}$ is defined to be 0 , and the generation of a child is 1 plus that of the father. In Xu et al. (2009) we proved that all triangles in a compatible patch $\omega_{e}$ have the same generation, which can be used to define the generation, gen $(\cdot)$, for a compatible bisection $b_{e}$ and the corresponding new vertex. For two different compatible bisections, $b_{e_{1}}$ and $b_{e_{2}}$, with the same generation, their patch are disjointed, i.e., $\omega_{e_{1}} \cap \omega_{e_{2}}=\varnothing$.

Lemma 2 (Stable Decomposition). For any $u, v \in \mathbb{K}$, the decompositions $u=\sum_{i=0}^{m} u_{i}, v=\sum_{i=0}^{m} v_{i}$ given by (9) satisfy

$$
\left(\sum_{i=0}^{m}\left\|u_{i}-v_{i}\right\|^{2}\right)^{\frac{1}{2}} \leq C\left(1+\left|\log h_{\min }\right|\right)\|u-v\| ;
$$

Proof. First note that the support of $\mathbb{V}_{i}$ is restricted to the extended patch $\widetilde{\omega}_{i, p_{i}}:=\cup_{x \in \omega_{i, p_{i}}} \omega_{i, x}$. Using inverse inequality and stability of $Q_{i}$, we have

$$
\left\|u_{i}-v_{i}\right\|_{\widetilde{\omega}_{i, p_{i}}}^{2} \leq C\left\|h_{\tau}^{-1}\left(u_{i}-v_{i}\right)\right\|_{L^{2}\left(\widetilde{\omega}_{i, p_{i}}\right)}^{2} \leq C\left(1+\left|\log h_{\min }\right|\right)\|u-v\|_{\widetilde{\omega}_{i, p_{i}}}^{2} .
$$

For bisections with the same generation $k$, the extended patches, $\widetilde{\omega}_{i, p_{i}}$, have finite overlapping and $\cup_{p, \operatorname{gen}(p)=k} \widetilde{\omega}_{i, p_{i}} \leq C|\Omega|$. Let $L=\max _{\tau \in \mathcal{T}} \operatorname{gen}(\tau)$. Then

$\sum_{i=1}^{m}\left\|u_{i}-v_{i}\right\|_{\widetilde{\omega}_{i, p_{i}}}^{2}=\sum_{k=1}^{L} \sum_{p_{i}, \operatorname{gen}\left(p_{i}\right)=k}\left\|u_{i}-v_{i}\right\|_{\widetilde{\omega}_{i, p_{i}}}^{2} \leq C L\left(1+\left|\log h_{\min }\right|\right)\|u-v\|_{\Omega}^{2}$.

The result then follows from the observation that $L \leq C\left|\log h_{\min }\right|$.

The proof of the following Strengthened Cauchy-Schwarz (SCS) inequality can be found in Xu et al. (2009). The idea of the proof is to apply standard SCS for each compatible decomposition and then rearrange the sum by generations. 
Lemma 3 (Strengthened Cauchy Schwarz Inequality). For any $u_{i}, v_{i} \in$ $\mathbb{V}_{i}, i=0, \ldots, m$, we have

$$
\left|\sum_{i=0}^{m} \sum_{j=0}^{m}\left(\nabla u_{i}, \nabla v_{j}\right)\right| \leq C\left(\sum_{i=0}^{m}\left|u_{i}\right|_{1}^{2}\right)^{1 / 2}\left(\sum_{i=1}^{m}\left|v_{i}\right|_{1}^{2}\right)^{1 / 2} .
$$

Applying the abstract theory (Theorem 3) and Lemma 2 and Lemma 3, we get the following rate of convergence.

Theorem 5 (Convergent Rate) Let $u^{k}$ be the $k$-th iteration of Algorithm 1 with the decomposition (9). We then have the following convergence rate

$$
\mathcal{J}\left(u^{k}\right)-\mathcal{J}\left(u^{*}\right) \leq C\left(1-\frac{1}{1+\left|\log h_{\min }\right|^{2}}\right)^{k} .
$$

\section{Numerical Experiments}

In this section, we use a numerical example by Tai (2003) to test the proposed algorithm: Let $\Omega=(-2,2)^{2}, f=0$ and the obstacle $\chi(x)=\sqrt{1-|x|^{2}}$ if $|x| \leq 1$ and -1 , otherwise. In this case, the exact solution is known to be

$$
u_{*}(x)= \begin{cases}\sqrt{1-|x|^{2}} & \text { if }|x| \leq r_{*} \\ -r_{*}^{2} \ln (|x| / 2) \sqrt{1-r_{*}^{2}} & \text { otherwise }\end{cases}
$$

where $r_{*} \approx 0.6979651482$. We give the Dirichlet boundary condition according to the exact solution above.

\begin{tabular}{|c|c|c|c|}
\hline Adaptive mesh & Degrees of freedom & $h_{\text {min }}$ & Reduction factor \\
\hline \hline 1 & 719 & $1.563 \mathrm{e}-2$ & 0.508 \\
2 & 1199 & $1.105 \mathrm{e}-2$ & 0.599 \\
3 & 2107 & $7.813 \mathrm{e}-3$ & 0.660 \\
4 & 3662 & $5.524 \mathrm{e}-3$ & 0.651 \\
5 & 6560 & $3.901 \mathrm{e}-3$ & 0.691 \\
6 & 11841 & $2.762 \mathrm{e}-3$ & 0.701 \\
\hline
\end{tabular}

Table 1. The reduction factors for the CDM algorithm on adaptively refined meshes. The reduction factor is the ratio of energy error between two consecutive iterations.

The contraction factors are computed and reported in Table 1 for a sequence of adaptive meshes, where the adaptive mesh refinement is driven by a posteriori error estimators starting from a uniform initial mesh; such adaptive algorithms and estimators can be found in Siebert and Veeser (2007) for example. The linear convergence rate is confirmed by our numerical experiments and the reduction rate is evaluated when the convergence becomes linear; there is a superlinear region in the beginning. 


\section{References}

Lori Badea, Xue-Cheng Tai, and Junping Wang. Convergence rate analysis of a multiplicative schwarz method for variational inequalities. SIAM Journal on Numerical Analysis, 41(3):1052-1073, 2003.

J. H. Bramble, J. E. Pasciak, and A. H. Schatz. The construction of preconditioners for elliptic problems by substructuring, I. Mathematics of Computation, 47:103-134, 1986.

Long Chen and Chen-Song Zhang. A coarsening algorithm and multilevel methods on adaptive grids by newest vertex bisection. Submitted to Journal of Computational Mathematics, 2009.

Long Chen, Ricardo H. Nochetto, and Jinchao Xu. Local multilevel methods on graded bisection grids for $H^{1}$ system. Submitted to Journal of Computational Mathematics, 2009.

C.W. Cryer. Successive overrelaxation methods for solving linear complementarity problems arising from free boundary problems. Proceedings of intensive seminary on free boundary problems, Pavie, Ed. Magenes, 1979.

C. Graser and R. Kornhuber. Multigrid methods for obstacle problems. Journal of Computational Mathematics, 27(1):1-44, 2009.

Ralf Kornhuber. Adaptive monotone multigrid methods for nonlinear variational problems. Advances in Numerical Mathematics. B. G. Teubner, Stuttgart, 1997.

R.H. Nochetto, K.G. Siebert, and A. Veeser. Theory of adaptive finite element methods: an introduction. In Ronald A. DeVore and Angela Kunoth, editors, Multiscale, Nonlinear and Adaptive Approximation. Springer, 2009.

Kunibert G. Siebert and Andreas Veeser. A unilaterally constrained quadratic minimization with adaptive finite elements. SIAM Journal on Optimization, 18(1):260-289, 2007.

Xue-Cheng Tai. Rate of convergence for some constraint decomposition methods for nonlinear variational inequalities. Numerische Mathematik, 93(4): 755-786, 2003.

Xue-Cheng Tai and Jinchao Xu. Global convergence of subspace correction methods for convex optimization problems. Mathematics of Computation, 71(237):105-124, 2002.

Xue-Cheng. Tai, B. Heimsund, and Jinchao. Xu. Rate of convergence for parallel subspace correction methods for nonlinear variational inequalities. In Domain decomposition methods in science and engineering (Lyon, 2000), Theory Eng. Appl. Comput. Methods, pages 127-138. Internat. Center Numer. Methods Eng. (CIMNE), Barcelona, 2002.

J. Xu, L. Chen, and R.H. Nochetto. Optimal multilevel methods for H(grad), $\mathrm{H}$ (curl), and $\mathrm{H}(\mathrm{div})$ systems on adaptive and unstructured grids. In Ronald A. DeVore and Angela Kunoth, editors, Multiscale, Nonlinear and Adaptive Approximation. Springer, 2009.

Jinchao $\mathrm{Xu}$. Iterative methods by space decomposition and subspace correction. SIAM Review, 34:581-613, 1992. 